\title{
Phase-space distribution functions for photon propagation in waveguides coupled to a qubit
}

\author{
O. O. Chumak* and E. V. Stolyarov \\ Institute of Physics of the National Academy of Sciences \\ pr. Nauki 46, Kyiv-28, MSP 03028 Ukraine
}

July 7, 2018

\begin{abstract}
We investigate propagation of few-photon pulses in waveguides coupled to a two-level system by means of the method of distribution functions in coordinate-momentum space that provides a detailed description of photon systems. We find that the distribution function of the transmitted pulse can be negative for the nonclassical input (i.e., single-photon Fock state). This reveals the quasiprobability nature of photon distribution. Analytical expressions for photon densities in the momentum space as well as in the coordinate space are obtained for the mentioned single-photon Gaussian input. We also study evolution of the multimode coherent-state input for an arbitrary photon number. Time-dependent differential equations describing average densities and fluctuations of outgoing photons are derived and solved. Influence of the number of input photons, pulse width, and radiation-atom interaction strength on the statistical properties of the fluctuations is investigated.
\end{abstract}

\footnotetext{
${ }^{*}$ Corresponding author: chumak@iop.kiev.ua
} 


\section{INTRODUCTION}

Traditionally the model of two-level system (TLS) is used to describe the interaction of electromagnetic radiation with atoms [1]. This model is quite reasonable if radiation frequency and transition frequency of the corresponding two levels are very close to each other. It was suggested to utilize Rydberg atoms controlled by electromagnetic fields as qubits in quantum information technologies [2]. There are other implementations of the qubits. Trapped ions [3], [4], semiconductor quantum dots [5], superconducting Josephson junctions can be used for this purpose. Qubits based on the Josephson junctions are recognized now to be the most promising for realization of quantum information processing devices (see, for example, Refs. [6]-[8]). There is a technological opportunity to couple qubits via transmission lines. Individual photons can act as transmitters of quantum states between remote qubits.

The aforesaid illustrates motivations to study TLS coupled to transmission lines. Atoms having a large dipole moment (Rydberg atoms) as well as transmission lines (including optical waveguides) that concentrate radiation energy in small volumes are used to increase the coupling. Strong interaction is desired for many applications whose aim is to achieve effective influence of one subsystem on the other. At the same time increase of interaction results in more pronounced nonlinearity of the system. This complicates theoretical analysis. Therefore many theoretical results were obtained only numerically (see, for example, [9]-[1]]). Fortunately, analysis can be simplified considerably for some particular states of the system. First of all, a single-photon Fock state of the incident radiation should be mentioned (see, for example, recent papers [12-[14]). Also things get simplified if the incident light is in a coherent state. For example, Ref. [14] deals with radiation which is initially in a single-mode coherent state. Much earlier paper [15] considers more general and more important for applications multimode coherent-state pulses. Results of Ref. [15] gives a possibility not only to obtain the reflectance and transmittance of a wave packet but also to study spatial structure of outgoing radiation and its dependence on the incident pulse shape. Moreover, paper [15] describes effective photon-photon "interaction" induced by coupling of the radiation with atoms.

Different formalisms are used in the cited papers. Method of scattering matrix [16] which is equivalent to the input-output formalism [17] is applied to photon scattering by TLS in [14]. Authors of [15] use an alternative approach based on calculation of Poynting vectors to study similar physical 
systems.

Recently we have applied method of photon phase-space distribution function to the problem of light propagation in the Earth atmosphere [18], [19]. In the present paper we use this method for description of light propagation in waveguides. We obtain a spatial structure and spectrum of the transmitted and reflected radiation which are useful for design of radiation with desired properties. Besides we analyze physical nature of the phase-space distribution functions. It is shown that they, like $Q-$ or $W$-distributions, can be negative for some specific parameters of the incident radiation.

Also equations describing fluctuations of outgoing photons are derived and solved. We show that the variance of the reflected radiation may be essentially lower than that of a coherent-state pulse. Thus, few-photon pulses with favorable statistical properties can be generated in course of radiationTLS interaction.

In the next Section, one-dimensional distribution functions are defined in terms of creation and annihilation operators of waveguide modes. The standard Hamiltonian describing light propagation and interaction with TLS is used to derive evolution equations.

\section{HAMILTONIAN AND PHOTON DISTRI- BUTION FUNCTIONS}

We consider a model Hamiltonian describing a two-level atom coupled to a single-polarization waveguide. The waveguide modes are assumed to form a one-dimensional continuum. Then the Hamiltonian is given by $(\hbar=1)$

$$
H=\int d k\left(\omega_{k}^{l} l_{k}^{\dagger} l_{k}+\omega_{k}^{r} r_{k}^{\dagger} r_{k}\right)+\frac{\omega_{a}}{2} \sigma_{z}+g \int d k\left[\sigma_{+}\left(l_{k}+r_{k}\right)+\left(l_{k}^{\dagger}+r_{k}^{\dagger}\right) \sigma_{-}\right]
$$

where $l_{k}$ and $r_{k}$ are the annihilation operators of photons propagating from the left side to the right side and vice versa, respectively. Photon frequencies are denoted correspondingly by $\omega_{k}^{l, r}$. Notations $l_{k}^{\dagger}$ and $r_{k}^{\dagger}$ stand for the creation operators. For a symmetric waveguide the linearized in $k$ dispersions in the vicinity of $\omega^{l, r}=\omega_{0}$ are given by $\omega_{k}^{l, r}=\omega_{0} \pm v k$ where $v$ and $-v$ $(v>0)$ are velocities of waves propagating from the left and from the right, respectively. Atomic operators $\sigma_{z}$ and $\sigma_{ \pm}$are defined by Pauli matrices: $\sigma_{ \pm}=\frac{1}{2}\left(\sigma_{x} \pm i \sigma_{y}\right), \sigma_{+} \sigma_{-}=\left(\sigma_{z}+1\right) / 2$. 
Field variables follow the usual bosonic commutation rules

$$
\left[l_{k}, l_{k^{\prime}}^{\dagger}\right]=\left[r_{k}, r_{k^{\prime}}^{\dagger}\right]=\delta\left(k-k^{\prime}\right),
$$

while the rest of commutators vanish. Also, field variables commute with atomic variables.

The first term in the right side of Eq. 1 describes electromagnetic field in the waveguide. The second term is the Hamiltonian of a two-level atom with transition frequency $\omega_{a}$. The third term describes the radiation-atom interaction whose strength is determined by parameter $g$. It is assumed that the atom is positioned at the origin of the coordinate system which makes the Hamiltonian to be independent explicitly of the atom coordinate. The interaction is presented in the rotating-wave approximation. Recently an approach which is free of this widely used constraint is developed in Ref. [20].

Photons moving from the left can be described by their density in the phase space $(x, q$-space). The corresponding function is defined as

$$
f^{l}(x, q, t)=\frac{1}{2 \pi} \int d k e^{-i k x} l_{q+k / 2}^{\dagger} l_{q-k / 2},
$$

where all operators are given in the Heisenberg picture. The distribution function (2) is defined by analogy with the 3D case (see more details in Ref. [21]). By integrating (21) over $x$ we obtain the density of $l$-photons in the momentum space:

$$
\hat{n}^{l}(q, t) \equiv \int d x f^{l}(x, q, t)=l_{q}^{\dagger}(t) l_{q}(t) .
$$

Accounting for the linear dependence $\omega^{l}(k)$ we can conclude that the average value $\left\langle\hat{n}^{l}(q, t)\right\rangle$ determines the spectral distribution of photons moving from the left. The spectrum can be obtained from Eq. (3) by changing $q \rightarrow$ $\left(\omega-\omega_{0}\right) / v$.

Similarly we can express the photon density in the coordinate space, $\hat{\rho}_{l}(x, t)$, in terms of the distribution function, $f^{l}(x, q, t)$, as:

$$
\hat{\rho}_{l}(x, t) \equiv \int d q f^{l}(x, q, t)=\frac{1}{2 \pi} \int d q d k e^{-i k x} l_{q+k / 2}^{\dagger} l_{q-k / 2} .
$$

Furthermore, by integrating $\hat{\rho}_{l}(x, t)$ over $x$ in the range of localization of the transmitted pulse we obtain the operator of total number of the transmitted 
photons, $N_{l}$, as

$$
\hat{N}_{l}(t)=\int d x \hat{\rho}_{l}(x, t)=\int d q l_{q}^{\dagger}(t) l_{q}(t)
$$

Expression (5) can be used for obtaining both transmittance and fluctuations of the transmitted photons.

Similar relationships for the $r$-photons follow from Eqs. (2)-(15) by replacing $l \rightarrow r$. Characteristics of the outgoing radiation depend on the initial state of the system and on the evolution of the above-mentioned operators.

\section{EVOLUTION EQUATIONS}

Evolution of the system variables is governed by a set of coupled Heisenberg equations

$$
\begin{gathered}
\left(\partial_{t}+i \omega_{q}^{l}\right) l_{q}=-i g \sigma_{-}, \\
\left(\partial_{t}+i \omega_{q}^{r}\right) r_{q}=-i g \sigma_{-}, \\
\left(\partial_{t}+i \omega_{a}\right) \sigma_{-}=i g \sigma_{z} \int d q\left(l_{q}+r_{q}\right), \\
\partial_{t} \sigma_{z}=-i 2 g \int d q\left[\sigma_{+}\left(l_{q}+r_{q}\right)-\left(l_{q}^{+}+r_{q}^{+}\right) \sigma_{-}\right] .
\end{gathered}
$$

Equations for variables $l_{q}^{\dagger}, r_{q}^{\dagger}, \sigma_{+}$can be obtained by Hermitian conjugation of Eqs. (66)-(8) .

Following Ref. [17] we represent a formal solution of Eq. (66) as

$$
l_{q}(t)=\tilde{l}_{q}(t)-i g \int_{t_{0}}^{t} d t^{\prime} e^{-i \omega_{q}^{l}\left(t-t^{\prime}\right)} \sigma_{-}\left(t^{\prime}\right)
$$

where $\tilde{l}_{q}(t)=l_{q}\left(t_{0}\right) e^{-i \omega_{q}^{l}\left(t-t_{0}\right)}$ and $t>t_{0}$. It is assumed that the pulse, localized at $t=t_{0}$ on the left from the atom, moves to the right. The first term in the right side of Eq. (10) describes the free-field propagation, while the second one represents the atom radiation. By integrating Eq. (10) over $q$, we obtain a useful relationship

$$
\int d q l_{q}(t)=\int d q \tilde{l}_{q}(t)-\frac{i \pi g}{v} \sigma_{-}(t)
$$


which is widely used in the literature. Then evolution of the atomic operators is governed by the equations

$$
\begin{gathered}
\left(\partial_{t}+i \omega_{a}+\Gamma / 2\right) \sigma_{-}=i g \sigma_{z} \int d q\left(\tilde{l}_{q}+\tilde{r}_{q}\right) \\
\left(\partial_{t}+\Gamma\right)\left(\sigma_{z}+1\right)=-i 2 g \int d q\left[\sigma_{+}\left(\tilde{l}_{q}+\tilde{r}_{q}\right)-\left(\tilde{l}_{q}^{+}+\tilde{r}_{q}^{+}\right) \sigma_{-}\right]
\end{gathered}
$$

where $\Gamma=4 \pi g^{2} / v$ and the tilde means the dependence on $t$ similar to the dependence $\tilde{l}_{q}(t)$. If we consider the tilded variables as given functions then we have a closed set of linear equations for obtaining atomic variables $\sigma_{ \pm}(t), \sigma_{z}(t)$. Using Eq. (12) we can exclude variables $\sigma_{ \pm}$from Eq. (13). When $\Gamma\left(t-t_{0}\right) \gg 1$ we get

$$
\begin{gathered}
\left(\partial_{t}+\Gamma\right)\left(\sigma_{z}+1\right) \\
=-2 g^{2} \int d q d k \int_{t_{0}}^{t} d t^{\prime}\left[e^{\left(i \omega_{a}-\Gamma / 2\right)\left(t-t^{\prime}\right)}\left(\tilde{l}_{k}^{\dagger}+\tilde{r}_{k}^{\dagger}\right)_{t^{\prime}} \sigma_{z}\left(t^{\prime}\right)\left(\tilde{l}_{q}+\tilde{r}_{q}\right)_{t}+H . c .\right] .
\end{gathered}
$$

The distribution function and the atom variables $\sigma_{ \pm}(t)$ are related by

$$
\begin{aligned}
f^{l}(x, q, t)= & \frac{1}{2 \pi} \int d k e^{-i k x}\left[\tilde{l}_{q+k / 2}^{\dagger}(t)+i g \int_{t_{0}}^{t} d t^{\prime} e^{i \omega_{q+k / 2}^{l}\left(t-t^{\prime}\right)} \sigma_{+}\left(t^{\prime}\right)\right] \\
& \times\left[\tilde{l}_{q-k / 2}(t)-i g \int_{t_{0}}^{t} d t^{\prime} e^{-i \omega_{q-k / 2}^{l}\left(t-t^{\prime}\right)} \sigma_{-}\left(t^{\prime}\right)\right] .
\end{aligned}
$$

Eq. (15) follows directly from Eqs. (2) and (10). By integrating over $q$, we obtain the expression for the photon density

$$
\hat{\rho}_{l}(x, t)=\tilde{\rho}_{l}(x, t)+\frac{\Gamma}{4 v} \Sigma_{t-x / v}+i \frac{g}{v} \int d q\left(\sigma_{+} \tilde{l}_{q}-\tilde{l}_{q}^{\dagger} \sigma_{-}\right)_{t-x / v}
$$

where $\Sigma \equiv \sigma_{z}+1, x>0$ and $\tilde{\rho}_{l}(x, t)$ is presented in terms of the "free" operators $\tilde{l}(t)^{\dagger}$ and $\tilde{l}(t)$. The reflected photons can be described by the operator

$$
\hat{\rho}_{r}(x, t)=\tilde{\rho}_{r}(x, t)+\frac{\Gamma}{4 v} \Sigma_{t+x / v}+i \frac{g}{v} \int d q\left(\sigma_{+} \tilde{r}_{q}-\tilde{r}_{q}^{\dagger} \sigma_{-}\right)_{t+x / v},
$$

where $x<0$ and $\tilde{\rho}_{r}(x, t)$ is defined via $\tilde{r}(t)^{\dagger}$ and $\tilde{r}(t)$. In what follows we will omit the term $\tilde{\rho}_{r}(x, t)$ because of the absence of photons propagating from the right at $t=t_{0}$. 


\section{SINGLE-PHOTON FOCK STATE}

We consider the simplest situation when the incident Gaussian wave packet contains only one photon distributed among waveguide modes. When this photon propagates from the left the single-photon Fock state can be defined as [1]

$$
\left|1_{l}\right\rangle=\frac{w^{1 / 2}}{\pi^{1 / 4}} \int d k e^{-i k x_{0}} e^{-k^{2} w^{2} / 2} l_{k}^{\dagger}\left(t_{0}\right)|0\rangle,
$$

where $|0\rangle$ is the vacuum state of the system. The coefficient before the integral is the normalization constant.

The average value of the initial distribution function is given by

$$
\left\langle 1_{l}\left|f^{l}\left(x, q, t_{0}\right)\right| 1_{l}\right\rangle=\frac{1}{\pi} e^{-\left(x-x_{0}\right)^{2} / w^{2}} e^{-q^{2} w^{2}} .
$$

It follows from Eq. (19) that $w$ can be interpreted as the width of a pulse centered at $x=x_{0}$. Moreover, the pulse spread in the momentum space, $\Delta q$, is of the order of $1 / w$. Before photons reach the ground-state atom their distribution function evolves as

$$
\left\langle 1_{l}\left|f^{l}(x, q, t)\right| 1_{l}\right\rangle=\frac{1}{\pi} e^{-X^{2}(t) / w^{2}} e^{-q^{2} w^{2}},
$$

where $X(t)=x-x_{0}-v\left(t-t_{0}\right)$. To obtain the average $\left\langle f^{l}(x, q, t)\right\rangle$ in the domain $v\left(t-t_{0}\right)>-x_{0}$ we should use its general form (15). Simple calculations result in the following average distribution function of the transmitted signal:

$$
\begin{gathered}
\left\langle 1_{l}\left|f^{l}(x, q, t)\right| 1_{l}\right\rangle=\frac{w}{2 \pi^{3 / 2}} \int d k e^{-i k X(t)} e^{-\left(q^{2}+k^{2} / 4\right) w^{2}} \\
\times\left[1-\frac{\Gamma}{2} \frac{\Gamma / 2+i k v}{\left(\omega_{a 0}-q v\right)^{2}-(k v-i \Gamma)^{2} / 4}\right],
\end{gathered}
$$

where $\omega_{a 0}=\omega_{a}-\omega_{0}$.

For obtaining Eq. (21) the relations

$$
\begin{gathered}
\sigma_{-}(t)\left|1_{l}\right\rangle=\left[\left\langle 1_{l}\right| \sigma_{+}(t)\right]^{\dagger} \\
=-i g\left(\frac{4 \pi}{w^{2}}\right)^{1 / 4} e^{-i \omega_{0}\left(t-t_{0}\right)} \int_{t_{0}}^{t} d t^{\prime} e^{-\left(i \omega_{a 0}+\Gamma / 2\right)\left(t-t^{\prime}\right)} e^{-\left[x_{0}+v\left(t^{\prime}-t_{0}\right)\right]^{2} / 2 w^{2}}|0\rangle
\end{gathered}
$$


are used. Also it is assumed that the atom has a sufficient time to relax to the ground state. It is so if

$$
v\left(t-t_{0}\right)+x_{0} \gg w+v / \Gamma .
$$

By integrating Eq. (21) over $x$ we get

$$
\left\langle\hat{n}^{l}(q, t)\right\rangle=\frac{w}{\pi^{1 / 2}} e^{-q^{2} w^{2}}\left[1-\frac{\Gamma^{2} / 4}{\left(\omega_{a 0}-q v\right)^{2}+\Gamma^{2} / 4}\right] .
$$

The second term in the square brackets describes resonant reflection of the

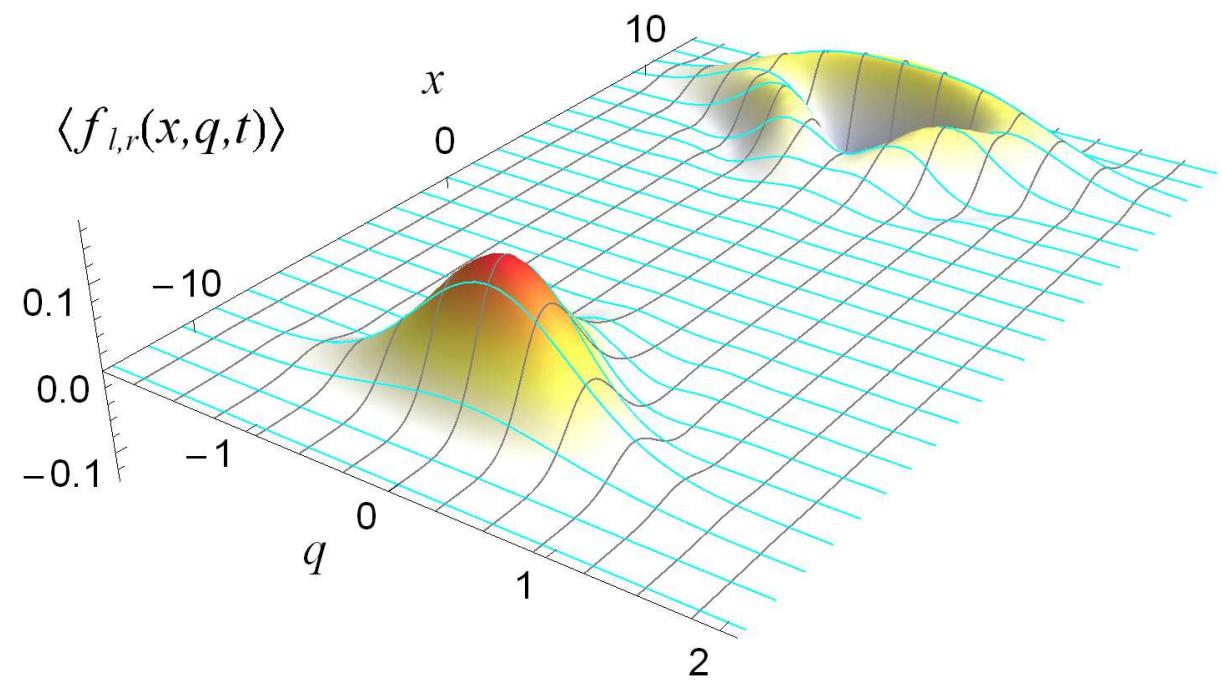

Figure 1: (Color online) Photon distributions $\left\langle f^{l}(x, q, t)\right\rangle(x>0)$ and $\left\langle f^{r}(x, q, t)\right\rangle(x<0)$ as functions of the phase-space variables, $x$ and $q$. Calculations are performed for $t=20, t_{0}=0, \Gamma=1, \omega_{a 0}=0$, and $x_{0}=-10$. Quantities $x, q, t$, and $\Gamma$ are given in units of $w$ (pulse width), $w^{-1}$ (inverse pulse width), $w / v$ (pulse duration), and $v / w$ (inverse pulse duration), respectively.

waves. This process is efficient when $\omega_{a 0}-v q \equiv \omega_{a}-\omega_{q}^{l} \leq \Gamma / 2$. If $\omega_{a 0} \neq 0$ the spectrum of the transmitted field is asymmetric which is different from 
the spectrum of the incident radiation. In fact, Eq. (24) describes filtering properties of the atom. It is straightforward to generalize Eq. (24) for an arbitrary shape of the incident pulse.

The average photon density is

$$
\left\langle\hat{\rho}_{l}(x, t)\right\rangle=\frac{1}{\pi^{1 / 2} w}\left|e^{-X^{2}(t) / 2 w^{2}}-\frac{i \Gamma w}{2^{3 / 2} \pi^{1 / 2}} \Phi[X(t)]\right|^{2},
$$

where

$$
\Phi(x)=\int d q \frac{e^{-i q x-q^{2} w^{2} / 2}}{\omega_{a 0}-q v+i \Gamma / 2} .
$$

For a very short incident pulse, $(\Gamma w / 2 v)<<1$, contribution of the term with $\Phi$ is negligible regardless of the value of $\omega_{a 0}$. This means that reflection is small for this case.

In the opposite case, $(\Gamma w / 2 v) \gg 1$, we obtain the photon density

$$
\left\langle\hat{\rho}_{l}(x, t)\right\rangle=\frac{1}{\pi^{1 / 2} w} e^{-X^{2}(t) / w^{2}}\left|1-\left(1-i 2 \omega_{a 0} / \Gamma\right)^{-1}\right|^{2}
$$

which is equal to zero when $\omega_{a 0}=0$. Hence, this is the case of full reflection. Nevertheless, for large detuning, $2 \omega_{a 0} / \Gamma \gg 1$, the radiation-atom interaction vanishes resulting in almost full transmission.

The distribution function of the reflected radiation is given by

$$
\left\langle 1_{l}\left|f^{r}(x, q, t)\right| 1_{l}\right\rangle=\frac{\Gamma^{2} w}{8 \pi^{3 / 2}} \int d k e^{-i k X^{r}(t)} \frac{e^{-\left(q^{2}+k^{2} / 4\right) w^{2}}}{\left(\omega_{a 0}+q v\right)^{2}-(k v+i \Gamma)^{2} / 4},
$$

where $X^{r}(t)=x+x_{0}+v\left(t-t_{0}\right)$.

Typical distribution functions, $\left\langle f^{l, r}(x, q, t)\right\rangle$, are shown in Fig. 1. In contrast to the initial positive distribution (19) the region with negative values of $\left\langle f^{r}(x, q, t)\right\rangle$ can be seen here that indicates a nonclassical nature of the reflected radiation. Physical quantity $\left\langle f^{l, r}(x, q, t)\right\rangle$ can be interpreted as a quasiprobability rather than a probability of the photon distribution in the phase space.

Using Eq. 27] we obtain coordinate and momentum distributions of the reflected photons as

$$
\left\langle\hat{\rho}_{r}(x, t)\right\rangle=\frac{\Gamma^{2} w}{8 \pi^{3 / 2}}\left|\Phi\left[-X^{r}(t)\right]\right|^{2}, \quad\left\langle\hat{n}^{r}(q, t)\right\rangle=\frac{\Gamma^{2} w}{4 \pi^{1 / 2}} \frac{e^{-q^{2} w^{2}}}{\left(\omega_{a 0}+q v\right)^{2}+\Gamma^{2} / 4} .
$$




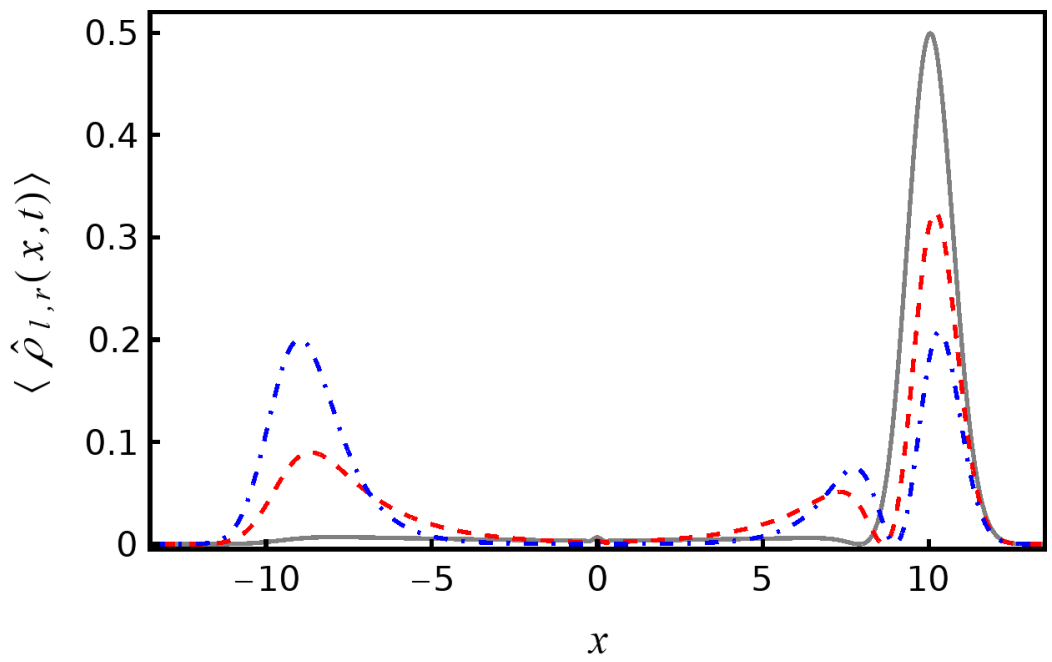

Figure 2: (Color online) Photon density in the configuration-space. Gray, red dashed, and blue dot-dashed curves are shown for $\Gamma$ equal to $0.1,0.5$, and 1, respectively. Other parameters are as in Fig. 1.

The second expression in (28) shows the resonant character of the reflection at $\omega_{a}-\omega_{q}^{r} \sim \Gamma / 2$.

As we see from Eqs. (24), (25) and (28) there are no regions with negative values of photon distributions $\left\langle\hat{\rho}_{r, l}(x, t)\right\rangle$ and $\left\langle\hat{n}^{r, l}(q, t)\right\rangle$. A set of curves in Figs. 2, 3 shows the expected tendency: reflection is bigger for stronger interaction.

Asymmetry of curves with respect to the central point, $q=0$, is seen in Figs. 3c and 3d. This is because only the incident photons with $q>0$ can be in resonance with the atom. Hence, they have the biggest probability to be reflected thus forming pronounced minima in $\left\langle\hat{n}^{l}(q, t)\right\rangle$ curves and the corresponding maxima in $\left\langle\hat{n}^{r}(q, t)\right\rangle$ curves. In the case of negative values of $\omega_{a o}$ similar plots can be obtained by formal replacement $q \rightarrow-q$ in Figs. 3c and $3 \mathrm{~d}$. 

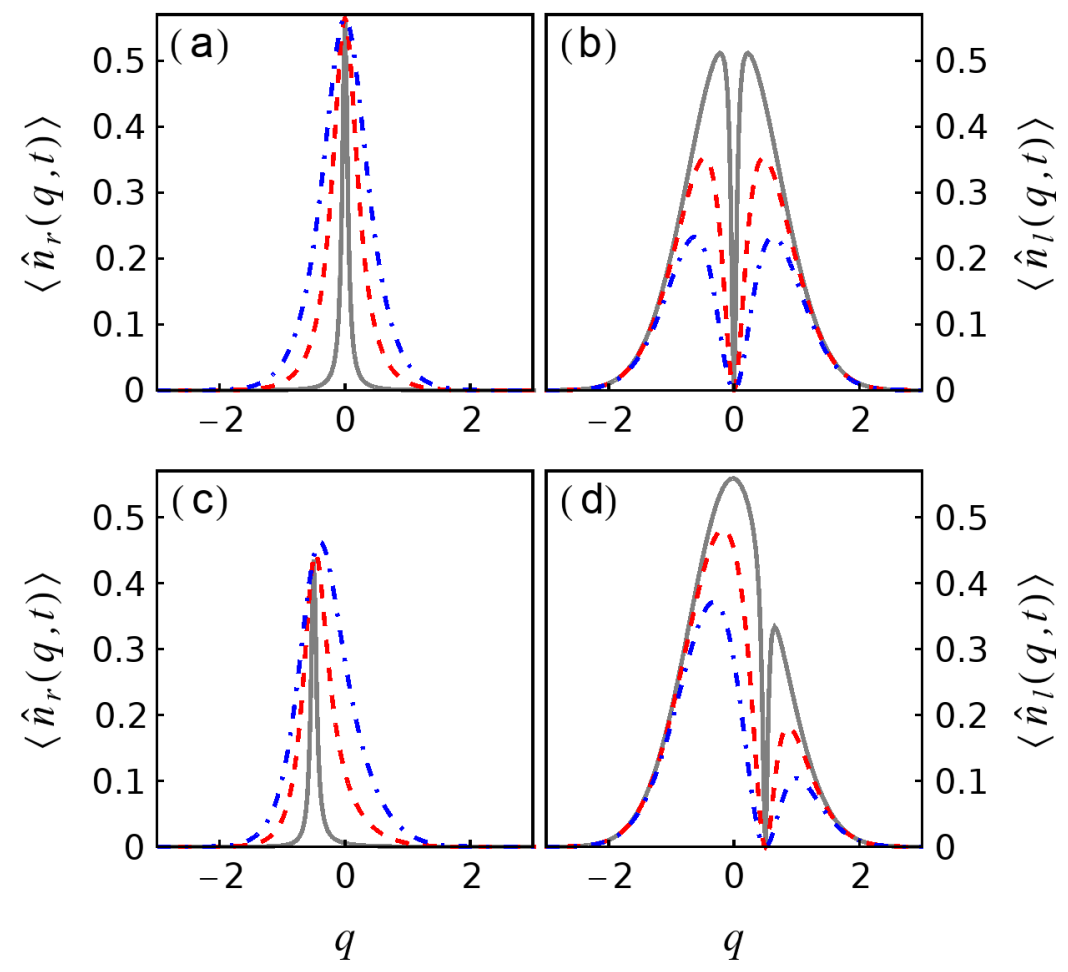

Figure 3: (Color online) Photon distribution in the momentum space. $\omega_{a 0}=$ $0(\mathrm{a}, \mathrm{b}) ; \omega_{a 0}=0.5(\mathrm{c}, \mathrm{d}) . \omega_{a 0}$ is given in units of $v / w$. Other notations are as in Figs. 1,2.

\section{COHERENT-STATE OF THE INCIDENT RADIATION}

Incident Gaussian pulse can be represented by a coherent-state wave packet. Following the paper [22] we define the corresponding wave function as

$$
|\Psi\{\alpha\}\rangle=\exp \left\{\int d k\left[\alpha_{k} l_{k}^{\dagger}-\alpha_{k}^{*} l_{k}\right]\right\}|0\rangle,
$$

where

$$
\alpha_{k}=\pi^{-1 / 4}\left(N_{0} w\right)^{1 / 2} e^{-i k x_{0}-k^{2} w^{2} / 2} .
$$

It can be easily verified that function (29) is the eigenfunction of all annihilation operators: $l_{k}|\Psi\{\alpha\}\rangle=\alpha_{k}|\Psi\{\alpha\}\rangle$. We use this property in further 
analysis.

By averaging the initial distribution function over the state (29) we obtain

$$
\left\langle\Psi\{\alpha\}\left|f^{l}\left(x, q, t_{0}\right)\right| \Psi\{\alpha\}\right\rangle=\frac{N_{0}}{\pi} e^{-\left(x-x_{0}\right)^{2} / w^{2}} e^{-q^{2} w^{2}},
$$

which is very similar to Eq. (19). The only free parameter, $N_{0}$, equal to the average number of photons per pulse, differs Eq. (30) from Eq. (19).

We use Eq. (15) to study photon density of the reflected and transmitted radiation. Integrating Eq. (15) over $q$ and using Eq. (14), the average density of photons, $\hat{\rho}_{l}(x, t)$ is obtained as

$$
\left\langle\hat{\rho}_{l}(x, t)\right\rangle=\left\langle\tilde{\rho}_{l}(x, t)\right\rangle-\frac{\Gamma}{4 v}\langle\Sigma\rangle_{t-x / v}-\frac{1}{2 v} \partial_{t}\langle\Sigma\rangle_{t-x / v}
$$

The last two terms in Eq. (31) describe atom response and interference of the response with the incoming field, respectively. A similar term for the backward-propagating pulse is given by

$$
\left\langle\hat{\rho}_{r}(x<0, t)\right\rangle=\frac{\Gamma}{4 v}\langle\Sigma\rangle_{t+x / v}
$$

Eq. (32) describes the radiation back-scattered by the atom. As we see the field distribution in the waveguide is expressed in terms of the average $\langle\Sigma\rangle$ which describes an atomic state. After averaging (14) over the initial wave function, $|\Psi\{\alpha\}\rangle$, we get

$$
\left(\partial_{t}+\Gamma\right)\langle\Sigma\rangle_{t}=-4 g^{2} p(t) \int_{t_{0}}^{t} d t^{\prime} e^{-\Gamma\left(t-t^{\prime}\right) / 2} p\left(t^{\prime}\right)\left\langle\sigma_{z}\right\rangle_{t^{\prime}} \cos \left[\omega_{a 0}\left(t-t^{\prime}\right)\right]
$$

where $p(t)=\pi^{1 / 4}\left(\frac{2 N_{0}}{w}\right)^{1 / 2} \exp \left\{-\left[x_{0}+v\left(t-t_{0}\right)\right]^{2} / 2 w^{2}\right\}$. Eq. (倓) should be completed with the initial condition $\langle\Sigma\rangle_{t_{0}}=0$.

The integro-differential equation (33) can be transformed into a differential equation. We consider the simplest case of $\omega_{a 0}=0$. Applying operator $\partial_{t}$ to both parts of Eq. (33) we obtain

$$
\hat{L}_{t}\langle\Sigma\rangle=4 g^{2} p^{2}(t)
$$

where

$$
\hat{L}_{t}=\partial_{t}^{2}+\left[\frac{3}{2} \Gamma+\left(t-t_{e}\right) \frac{v^{2}}{w^{2}}\right] \partial_{t}+\left[\frac{\Gamma^{2}}{2}+\left(t-t_{e}\right) \frac{v^{2}}{w^{2}} \Gamma+4 g^{2} p^{2}(t)\right]
$$




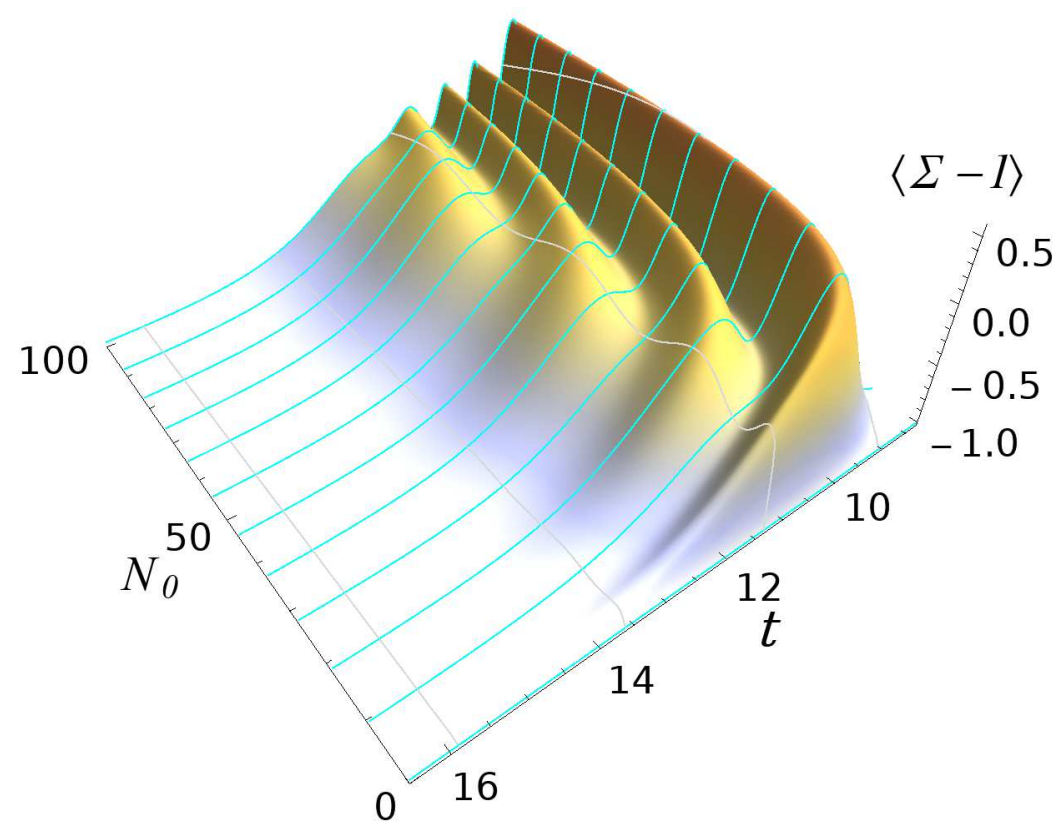

Figure 4: (Color online) Atom excitation dynamics vs initial photon number $N_{0}$ for $\Gamma=1$.

and $t_{e}=t_{0}+\left|x_{0}\right| / v$.

In the case of a long pulse, $(\Gamma w / 2 v) \gg 1$, the quasistationary state of $\langle\Sigma\rangle$ given by

$$
\langle\Sigma\rangle_{q s} \approx\left(1+\Gamma^{2} / 8 g^{2} p^{2}(t)\right)^{-1},
$$

can be realized. It follows from the above expression that for large (small) driving fields, $p^{2}(t) \rightarrow \infty\left(p^{2}(t) \rightarrow 0\right)$, the value of $\langle\Sigma\rangle_{q s}$ is equal to $1(0)$ in agreement with the simplest qualitative reasonings. There are damped oscillations of $\Sigma$ around this state. Their evolution is governed by Eq. (34) which in the long-pulse limit reduces to

$$
\delta \ddot{\Sigma}+\delta \dot{\Sigma} \frac{3}{2} \Gamma+\delta \Sigma\left[\frac{\Gamma^{2}}{2}+4 g^{2} p^{2}(t)\right]=0,
$$

where $\delta \Sigma=\langle\Sigma\rangle-\langle\Sigma\rangle_{q s}$. Ignoring the dependence of $p$ on $t$ we seek a solution in the form $\delta \Sigma \sim e^{\lambda t}$. Then the equation for $\lambda$ is given by

$$
\lambda^{2}+\lambda \frac{3}{2} \Gamma+\frac{\Gamma^{2}}{2}+4 g^{2} p^{2}(t)=0
$$


It follows from Eq. (36) that oscillations of $\langle\Sigma(t)\rangle$ arise if only $(2 v p / \pi g)>$ 1 ,oscillation decay rate with the oscillation decay rate of the order of $3 \Gamma / 4$.
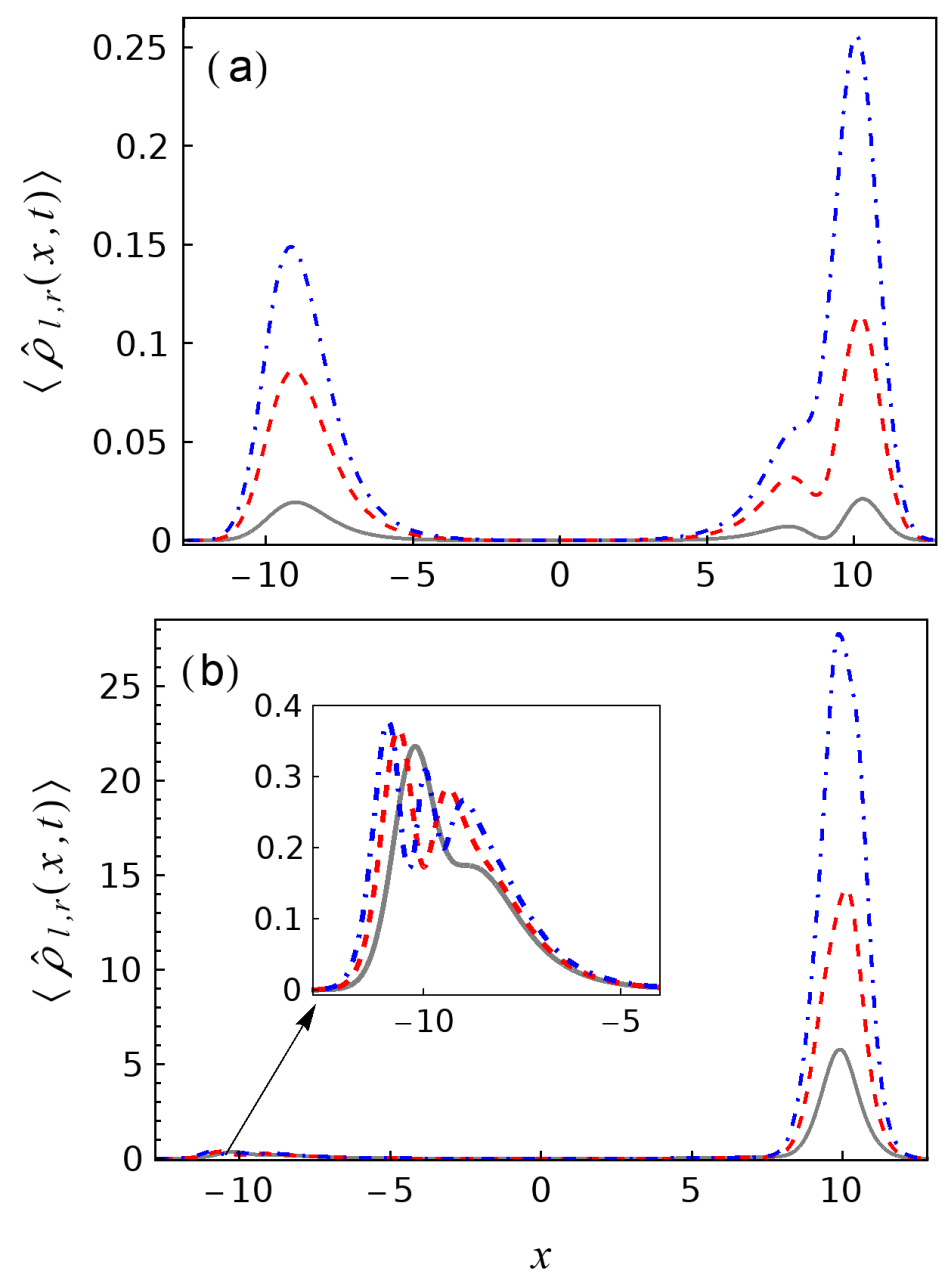

Figure 5: (Color online) Photon configuration-space densities: (a) $N_{0}=0.1$ - gray solid line, $N_{0}=0.5$ - red dashed line, $N_{0}=1$ - blue dash-dot line; (b) $N_{0}=10$ - gray solid line, $N_{0}=25$ - red dashed line, $N_{0}=50$ - blue dash-dot line (reflected pulses are shown in the inset). Input radiation is in coherent state, $\Gamma=1$ for all curves.

Oscillating behavior of the atom excitations (Rabi oscillations) can be also seen in Fig. 4 which illustrate typical solutions of Eq. (34). The most 
pronounced oscillations are for larger values of $\Gamma$ and $N_{0}$.

The solution of Eq. (34) is also used to obtain a configuration-space densities of transmitted and reflected photons. The calculated data are shown in Fig. 5. Qualitatively, the curves are similar to those in Fig. 2 if $N_{0}$ is small (see Fig. 5a). Pronounced oscillations are present only for the reflected photons when $N_{0}$ is sufficiently large (see the inset in Fig. 5b).

The numbers of reflected and transmitted photons are obtained after integration of the photon densities, $\hat{\rho}_{r}$ and $\hat{\rho}_{l}$,

$$
\begin{gathered}
N_{r}=\left\langle\hat{N}_{r}\right\rangle=\int_{v\left(t_{0}-t\right)}^{0} d x\left\langle\hat{\rho}_{r}(x, t)\right\rangle=\frac{\Gamma}{4} \int_{t_{0}}^{t} d \tau\left\langle\Sigma_{\tau}\right\rangle, \\
N_{l}=\left\langle\hat{N}_{l}\right\rangle=\int_{0}^{v\left(t-t_{0}\right)} d x\left\langle\hat{\rho}_{l}(x, t)\right\rangle=\int_{t_{0}}^{t} d \tau v\left\langle\tilde{\rho}_{l}[v(t-\tau), t]\right\rangle-N_{r},
\end{gathered}
$$

where the conditions $\tilde{r}_{q}|\Psi\rangle=\langle\Psi| \tilde{r}_{q}^{\dagger}=0$ are used. Intervals for integration over $x$ are chosen to be sufficiently large to cover the regions where the particle densities differ from zero. Corresponding time interval, $t-t_{0}$, satisfies condition (23).

The term $-\partial_{t}\left\langle\sigma_{z}\right\rangle / 2 v$, which is important for determining the pattern of the transmitted pulse, does not contribute to the total number of the transmitted photons because of zero value of $\langle\Sigma\rangle$ at the boundary points $t$ and $t_{0}$. The calculated values of $N_{r}$ and $N_{l}$ are shown in Fig. 6 by dashed lines.

To estimate upper limit of the reflected photon number, $N_{r}$, the inequality $\langle\Sigma\rangle<2$ and Eq. (37) are used. Thus we have $N_{r} \leq \Gamma w /(2 v)$. The limiting value of $N_{r}$ does not depend on $N_{0}$ and can be small even if $N_{0}>>1$. Therefore the reflected radiation can be used as a controllable source of fewphoton pulses. Also reflected photons can be useful, for example, to check the atom state or obtain the interaction parameter $g$.

\section{FLUCTUATIONS OF OUTGOING PHO- TONS}

Since, according to Eqs. (13), (16) , and (17), $\hat{N}_{l}+\hat{N}_{r} \equiv \tilde{N}_{l}$, noise properties of the reflected and transmitted photons can be described by the following variances

$$
\left\langle\Delta \hat{N}_{r}^{2}\right\rangle \equiv\left\langle\left(\hat{N}_{r}-N_{r}\right)^{2}\right\rangle=\left\langle\hat{N}_{r}^{2}\right\rangle-N_{r}^{2},
$$




$$
\left\langle\Delta \hat{N}_{l}^{2}\right\rangle \equiv\left\langle\left(\hat{N}_{l}-N_{l}\right)^{2}\right\rangle=\left\langle\left(\tilde{N}_{l}-\hat{N}_{r}\right)^{2}\right\rangle-N_{l}^{2}
$$

where

$$
\hat{N}_{r}=\int_{t_{0}}^{t} d \tau\left[\frac{\Gamma}{4} \Sigma+i g \int d q\left(\sigma_{+} \tilde{r}_{q}-\tilde{r}_{q}^{\dagger} \sigma_{-}\right)\right]_{\tau} .
$$

Eqs. (39) and (40) represent the mean square deviations of the photon numbers from their average values $N_{r}$ and $N_{l}$. Since there are no reflected photons when $g=0$, the fluctuations of the transmitted photons are identical to those of the incident radiation:

$$
\left\langle\Delta \hat{N}_{l}^{2}\right\rangle=\left\langle\Delta \tilde{N}_{l}^{2}\right\rangle=N_{l}=N_{0}
$$

Eq. (42) can be easily verified using the explicit term (5) for $\hat{N}_{l}$ and Eq.
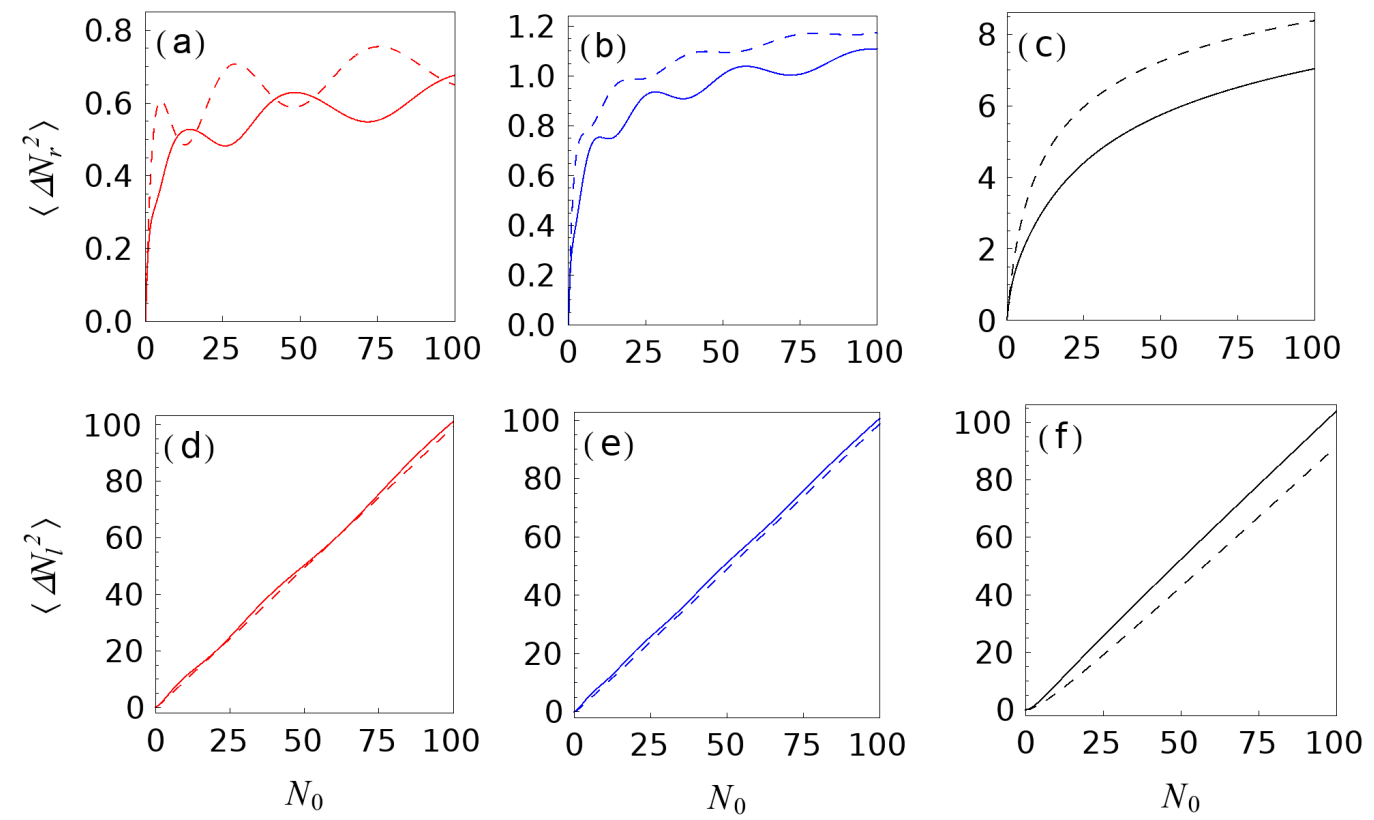

Figure 6: (Color online) Photon number variances. Solid lines - obtained using the definitions (39), (40) and numerical solutions of Eqs. (44) and (45). In the case of Poissonian statistics of outgoing radiation, the corresponding variances would be given by $N_{r, l}$ - shown by dashed lines. $\Gamma=0.5$ for $(\mathrm{a}, \mathrm{d})$; $\Gamma=1$ for $(\mathrm{b}, \mathrm{e}) ; \Gamma=10$ for $(\mathrm{c}, \mathrm{f})$.

(29) for the multimode coherent-state, $|\Psi\{\alpha\}\rangle$. 
In what follows we study the modification of the photon statistics caused by the radiation-atom interaction. To simplify further analysis we again consider only the resonant case $\omega_{0}=\omega_{a}$. As it is shown in the Appendix

$$
\left\langle\hat{N}_{r}^{2}\right\rangle=\frac{\Gamma^{2}}{8} \int_{t_{0}}^{t} d \tau \int_{t_{0}}^{\tau} d \tau^{\prime}\left\langle S\left(\tau, \tau^{\prime}\right)\right\rangle+N_{r},
$$

where $S\left(\tau, \tau^{\prime}\right)=2 \sigma_{+}\left(\tau^{\prime}\right) \Sigma(\tau) \sigma_{-}\left(\tau^{\prime}\right)$. It can be easily seen that the reflected photons does not obey the Poissonian statistics if the first term in the right side of Eq. (43) is bigger or smaller than $N_{r}^{2}$ (super- or sub-Poissonian statistics, respectively).

The average value $\left\langle S\left(\tau, \tau^{\prime}\right)\right\rangle$ is governed by the equation

$$
\hat{L}_{\tau}\left\langle S\left(\tau, \tau^{\prime}\right)\right\rangle=4 g^{2} p^{2}(\tau)\left\langle\Sigma\left(\tau^{\prime}\right)\right\rangle
$$

which can be derived similarly to Eq. (34). The definition of $\left\langle S\left(\tau, \tau^{\prime}\right)\right\rangle$ and properties of the Pauli matrices, namely $\sigma_{+}(\tau) \Sigma(\tau)=\Sigma(\tau) \sigma_{-}(\tau)=\left(\sigma_{+}\right)^{2}=$ $\left(\sigma_{-}\right)^{2}=0$, let us get the initial conditions for $\left\langle S\left(\tau, \tau^{\prime}\right)\right\rangle$ as $\left\langle S\left(\tau, \tau^{\prime}\right)\right\rangle=$ $\partial_{\tau}\left\langle S\left(\tau, \tau^{\prime}\right)\right\rangle=0$ when $\tau=\tau^{\prime}$.

After solving Eqs. (34) and (44), it becomes possible to calculate the mean square value $\left\langle\hat{N}_{r}^{2}\right\rangle$ of the reflected photons. In Fig. 6a crossovers from sub-Poissonian to super-Poissonian statistics are seen for some specific values of $N_{0}$. For bigger $\Gamma$ (see. Figs. 6b,c) the variances $\left\langle\Delta \hat{N}_{r}^{2}\right\rangle$ are characterized by sub-Poissonian statistics. Similar to results of Sect. 4 we see nonclassical nature of outgoing radiation that can be used in applications.

To obtain $\left\langle\Delta \hat{N}_{l}^{2}\right\rangle$ we should know not only $\left\langle\hat{N}_{r}^{2}\right\rangle$ but also $\left\langle\tilde{N}_{l} \hat{N}_{r}\right\rangle=$ $\frac{\Gamma}{4} \int_{t_{0}}^{t} d \tau\left\langle\tilde{N}_{l} \Sigma_{\tau}\right\rangle$. The quantity $\left\langle\tilde{N}_{l} \Sigma_{\tau}\right\rangle$ entering the integrand obeys the equation

$$
\hat{L}_{\tau}\left\langle\tilde{N}_{l} \Sigma(\tau)\right\rangle=4 g^{2} p^{2}(\tau)\left\langle N_{0}-\sigma_{z}(\tau)\right\rangle .
$$

Initial conditions are the same as for $\langle\Sigma(\tau)\rangle$, i.e.

$$
\left\langle\tilde{N}_{l} \Sigma\left(\tau=t_{0}\right)\right\rangle=\partial_{\tau}\left\langle\tilde{N}_{l} \Sigma\left(\tau=t_{0}\right)\right\rangle=0 .
$$

Fluctuations of the transmitted radiation are very similar to the fluctuations of the incident light (see Figs. 6d-f). This is due to saturation of the TLS response: only insignificant number of photons are involved in the atom excitation. Most photons are not affected by the atom and conserve the statistical properties of the coherent-state input. 


\section{Discussion and Conclusion}

The purpose of this paper is to analyze distinct features of outgoing radiation. These features describe not only spatial but also frequency distribution (i.e. spectrum) of the radiation. Therefore it is appropriate to use the method of photon distribution functions. Restricting our analysis to an incident pulse formed as Gaussian packet of single-photon Fock state it becomes possible to obtain analytical expressions for distribution functions of outgoing photons. In Fig. 1 one can see the change of sign of distribution function of the transmitted photons. This means that $\left\langle f^{r, l}(x, q, t)\right\rangle$ describes rather a quasiprobability than a probability of photon distribution.

Integrating $\left\langle f^{r, l}(x, q, t)\right\rangle$ over variables $q$ or $x$ we obtain spatial or frequency distributions, respectively. Spectra of transmitted and reflected radiation have very different structures strongly dependent on both the detuning, $\omega_{a 0}$, and the dimensionless parameter $\Gamma w / v$ (see Fig. 3).

The criterion of negligible reflection as well as the criterion of negligible transmission are derived using the explicit term for spatial distribution of photons, Eq. (25). These criteria and the data in Fig. 2 agree well with earlier studies in this field which show a higher probability for short pulses to be transmitted.

The case of coherent state of the incident radiation is also considered. The excitation-relaxation rates of the atom depend on the number of photons, $N_{0}$. For multi-photon pulses, $N_{0}>>1$, the Rabi frequency is proportional

to $N_{0}^{1 / 2}$. The tendency for oscillation frequency to grow with $N_{0}$ is seen in Fig. 4.

Numerical data in Figs. 4, 5 are obtained from solution of Eq. (34) which governs the evolution of $\langle\Sigma(t)\rangle \equiv\left\langle\sigma_{z}(t)\right\rangle+1$. The quantity $\langle\Sigma(t)\rangle$ describes the probability of TLS to be excited. At the same time, $\langle\Sigma(t)\rangle$ and its derivative $\partial_{t}\langle\Sigma(t)\rangle$ determine the photon densities $\left\langle\rho_{l, r}(x, t)\right\rangle$ [see Eqs. (31,32)]. The interconnection of these physical quantities is explained by energy conservation: each atom excitation is accompanied by annihilation of one photon in the waveguide and vice versa [see the interaction term in Eq. (1)].

In view of possible applications of outgoing radiation its noise characteristics are also important. Eqs. (40) and (43) and solutions of Eqs. (44,45) make it possible to calculate variances of photon numbers. It can be seen from Fig. 6 that in most cases the transmitted photons obey the super-Poissonian statistics, while the reflected photons obey the sub-Poissonian one that is in 
a qualitative consistence with results of Ref. [23] where bunching of transmitted photons and antibunching of reflected photons only were obtained. In our formalism, the case considered in [23] corresponds to infinitely long pulses. Hence, comparison of [23] with our data can be plausible for only large values of the parameter $\Gamma w / v$ used in Fig. 6 .

The approach used in the present paper can be easily modified to study more complex phenomena. Among of these, propagation of electromagnetic pulses in a waveguide, coupled to a pair of TLS, is of interest.

\section{Acknowledgment}

We thank V. Bondarenko and A. Sokolov for their interest to this research and stimulating discussions.

\section{Appendix: derivation of Eq. (43)}

It follows from Eq. (41) that the average $\left\langle\hat{N}_{r}^{2}\right\rangle$ is given by

$$
\begin{gathered}
\left\langle\hat{N}_{r}^{2}\right\rangle=\frac{\Gamma^{2}}{16} \int_{t_{0}}^{t} d \tau \int_{t_{0}}^{t} d \tau^{\prime}\left[\left\langle\Sigma(\tau) \Sigma\left(\tau^{\prime}\right)\right\rangle+\frac{i v}{\pi g} \int d q\left\langle\sigma_{+}\left(\tau^{\prime}\right) \tilde{r}_{q}\left(\tau^{\prime}\right) \Sigma(\tau)-\text { h.c. }\right\rangle\right. \\
\left.+\frac{4 v}{\pi \Gamma} \int d q \int d q^{\prime}\left\langle\sigma_{+}(\tau) \tilde{r}_{q}(\tau) \tilde{r}_{q^{\prime}}^{\dagger}\left(\tau^{\prime}\right) \sigma_{-}\left(\tau^{\prime}\right)\right\rangle\right]
\end{gathered}
$$

It is useful to represent the product $\tilde{r}_{q}(\tau) \tilde{r}_{q^{\prime}}^{\dagger}\left(\tau^{\prime}\right)$ in the ordered form as $\tilde{r}_{q}(\tau) \tilde{r}_{q^{\prime}}^{\dagger}\left(\tau^{\prime}\right)=\tilde{r}_{q^{\prime}}^{\dagger}\left(\tau^{\prime}\right) \tilde{r}_{q}(\tau)+\delta\left(q-q^{\prime}\right) \exp \left[i \omega_{q}^{r}\left(\tau^{\prime}-\tau\right)\right]$. The part of the last term in Eq. (46) that is proportional to $\delta\left(q-q^{\prime}\right)$ gives a contribution to $\left\langle\hat{N}_{r}^{2}\right\rangle$ equal to

$$
\frac{\Gamma}{4} \int_{t_{0}}^{t} d \tau\langle\Sigma(\tau)\rangle=N_{r} .
$$

The remaining part of the third term in Eq. (46),

$$
\int d q \int d q^{\prime}\left\langle\sigma_{+}(\tau) \tilde{r}_{q^{\prime}}^{\dagger}\left(\tau^{\prime}\right) \tilde{r}_{q}(\tau) \sigma_{-}\left(\tau^{\prime}\right)\right\rangle
$$


is equal to zero. To prove this let us consider the term $\int d q^{\prime}\langle\Psi| \sigma_{+}(\tau) \tilde{r}_{q^{\prime}}^{\dagger}\left(\tau^{\prime}\right)$ where $|\Psi\rangle$ is given by Eq. (29). Representing $\tilde{r}_{q^{\prime}}^{\dagger}\left(\tau^{\prime}\right)$ as

$$
\tilde{r}_{q^{\prime}}^{\dagger}(\tau) e^{i \omega_{q^{\prime}}^{r}\left(\tau^{\prime}-\tau\right)}=\left[r_{q^{\prime}}^{\dagger}(\tau)-i g \int_{t_{0}}^{\tau} d \tau^{\prime \prime} e^{i \omega_{q^{\prime}}^{r}\left(\tau-\tau^{\prime \prime}\right)} \sigma_{+}\left(\tau^{\prime \prime}\right)\right] e^{i \omega_{q^{\prime}}^{r}\left(\tau^{\prime}-\tau\right)}
$$

and taking into account that $r_{q^{\prime}}^{\dagger}(\tau)$ commutes with $\sigma_{+}(\tau)$, we have

$$
\begin{gathered}
\int d q^{\prime}\langle\Psi| \sigma_{+}(\tau) \tilde{r}_{q^{\prime}}^{\dagger}\left(\tau^{\prime}\right)=\int d q^{\prime}\langle\Psi|\left[r_{q^{\prime}}^{\dagger}(\tau) \sigma_{+}(\tau)\right. \\
\left.-i g \int_{t_{0}}^{\tau} d \tau^{\prime \prime} e^{i \omega_{q^{\prime}}^{r}\left(\tau-\tau^{\prime \prime}\right)} \sigma_{+}(\tau) \sigma_{+}\left(\tau^{\prime \prime}\right)\right] e^{i \omega_{q^{\prime}}^{r}\left(\tau^{\prime}-\tau\right)} \\
=i g \int d q^{\prime} \int_{t_{0}}^{\tau} d \tau^{\prime \prime} e^{i \omega_{q^{\prime}}^{r}\left(\tau^{\prime}-\tau^{\prime \prime}\right)}\langle\Psi|\left[\sigma_{+}\left(\tau^{\prime \prime}\right), \sigma_{+}(\tau)\right] \\
=i \frac{2 \pi g}{v}\langle\Psi|\left[\sigma_{+}\left(\tau^{\prime}\right), \sigma_{+}(\tau)\right] \theta\left(\tau-\tau^{\prime}\right) .
\end{gathered}
$$

The last expression in Eq. (50) is obtained after integration over $q^{\prime}$ and $\tau^{\prime \prime}$. Also, we use here the condition $\langle\Psi| \hat{r}_{q^{\prime}}^{\dagger}=0$.

The value of $\int d q \tilde{r}_{q}(\tau) \sigma_{-}\left(\tau^{\prime}\right)|\Psi\rangle$ can be obtained from Eq. (150) by means of Hermitian conjugation and replacement $\tau \longleftrightarrow \tau^{\prime}$ :

$$
\int d q \tilde{r}_{q}(\tau) \sigma_{-}\left(\tau^{\prime}\right)|\Psi\rangle=-i \frac{2 \pi g}{v}\left[\sigma_{-}\left(\tau^{\prime}\right), \sigma_{-}(\tau)\right]|\Psi\rangle \theta\left(\tau^{\prime}-\tau\right) .
$$

Inserting the last terms of Eqs. (150) and (51) into Eq. (48) we get zero.

A similar procedure is used to simplify the second term in the brackets of Eq. (46). Repeating the previous reasonings we obtain the following relations (see also the Appendix in Ref. [15]):

$$
\begin{aligned}
& \int d q \tilde{r}_{q}\left(\tau^{\prime}\right) \Sigma(\tau)|\Psi\rangle=i \frac{2 \pi g}{v}\left[\sigma_{-}\left(\tau^{\prime}\right), \Sigma(\tau)\right]|\Psi\rangle \theta\left(\tau-\tau^{\prime}\right), \\
& \int d q\langle\Psi| \Sigma(\tau) \tilde{r}_{q}^{\dagger}\left(\tau^{\prime}\right)=i \frac{2 \pi g}{v}\langle\Psi|\left[\sigma_{+}\left(\tau^{\prime}\right), \Sigma(\tau)\right] \theta\left(\tau-\tau^{\prime}\right) .
\end{aligned}
$$

Using Eqs. (52), (53) we have

$$
\frac{\Gamma^{2}}{16} \int_{t_{0}}^{t} d \tau \int_{t_{0}}^{t} d \tau^{\prime} \frac{i v}{\pi g} \int d q\left\langle\sigma_{+}\left(\tau^{\prime}\right) \tilde{r}_{q}\left(\tau^{\prime}\right) \Sigma(\tau)-\text { h.c. }\right\rangle
$$




$$
=-\frac{\Gamma^{2}}{16} \int_{t_{0}}^{t} d \tau \int_{t_{0}}^{\tau} d \tau^{\prime}\left\langle\Sigma(\tau) \Sigma\left(\tau^{\prime}\right)+\Sigma\left(\tau^{\prime}\right) \Sigma(\tau)-4 \sigma_{+}\left(\tau^{\prime}\right) \Sigma(\tau) \sigma_{-}\left(\tau^{\prime}\right)\right\rangle .
$$

Finally, the overall contribution of three terms in brackets of Eq. (46) results in Eq. (43).

\section{References}

[1] L. Mandel and E. Wolf, Optical coherence and quantum optics (Cambridge University Press, Cambridge, England, 1995).

[2] D. Jaksch, J. I. Cirac, P. Zoller, S. L. Rolston, R. Côté, and M. D. Lukin, Phys. Rev. Lett. 85, 2208 (2000).

[3] J. I. Cirac and P. Zoller, Phys. Rev. Lett. 74, 4091 (1995).

[4] C. Monroe, D. Leibfried, B.E. King, D. M. Meekhof, W. M. Itano, and D. J. Wineland, Phys. Rev. A 55, R24892491 (1997).

[5] F. Henneberger and O. Benson, Semiconductor Quantum Bits (Pan Stanford, Singapore, 2008).

[6] J. M. Martinis, S. Nam, J. Aumentado, K. M. Lang, and C. Urbina, Phys. Rev. B 67, 094510 (2003).

[7] A. Blais, R.S. Huang, A. Wallraff, S. M. Girvin, and R.J. Schoelkopf, Phys. Rev. A 69, 062320 (2004).

[8] A. Wallraff, D. I. Schuster, A. Blais, L. Frunzio, J. Majer, M. H. Devoret, S. M. Girvin, and R. J. Schoelkopf, Phys. Rev. Lett. 95, 060501(2005).

[9] G. Drobný, M. Havukainen, and V. Bužek, J. Mod. Opt. 47, 851 (2000).

[10] M. Havukainen, G. Drobný, S. Stenholm, and V. Bužek, J. Mod. Opt. 46, 1343 (1999).

[11] V. Bužek, G. Drobný, M. G. Kim, M. Havukainen, and P. L. Knight, Phys. Rev. A 60, 582 (1999).

[12] J.-T. Shen and S. Fan, Phys. Rev. A 79, 023837 (2009).

[13] J.-T. Shen and S. Fan, Phys. Rev. A 79, 023838 (2009). 
[14] S. Fan, S. E. Kocabaş, and J.-T. Shen, Phys. Rev. A 82, 063821 (2010).

[15] P. Domokos, P. Horak, and H. Ritsch, Phys. Rev. A 65, 033832 (2002).

[16] T. Shi and C.P. Sun, Phys. Rev. B 79, 205111 (2009).

[17] D. F. Walls and G. J. Milburn, Quantum Optics, 2nd ed. (Springer, New York, 2008).

[18] G. P. Berman and A. A. Chumak, Phys. Rev. A 74, 013805 (2006).

[19] G. P. Berman and A. A. Chumak, Proc. of SPIE 6710, 67100M-1 (2007).

[20] Yu. V. Bezvershenko and P. I. Holod, Phys. Lett. A375, 3936 (2011).

[21] O. Chumak and N. Sushkova, Ukr. J. Phys. 57, 30 (2012).

[22] K. J. Blow, R. Loudon, S. D. Phoenix, and T. J. Shepherd, Phys. Rev. A 42, 4102 (1990).

[23] S.E. Kocabaş, E. Rephaeli, and S. Fan, Phys. Rev. A 85, 023817 (2012). 